INTERNATIONAL UNION OF PURE

AND APPLIED CHEMISTRY

ANALYTICAL CHEMISTRY DIVISION

COMMISSION ON SEPARATION METHODS IN ANALYTICAL CHEMISTRY*

Project 530/10/95 (Revised title)

July 2001

\title{
TERMINOLOGY FOR ANALYTICAL CAPILLARY ELECTROMIGRATION TECHNIQUES
}

(IUPAC Recommendations 2001)

Prepared for Publication by

MARJA-LIISA RIEKKOLA ${ }^{1}$ AND JAN ÅKE JÖNSSON ${ }^{2}$

${ }^{1}$ Laboratory of Analytical Chemistry, Department of Chemistry, P.O. Box 55, FIN-00014 University of Helsinki, Finland

${ }^{2}$ Department of Analytical Chemistry, Lund University

P.O. Box 124, SE-221 00 Lund, Sweden

*Membership of the Commission during the period (1995-2001) when this report was prepared was as follows:

Chairman: A. Marton (Hungary, 1995-1999), R. M. Smith (UK, 2000-2001); Secretary: R. M. Smith (UK, $1995-$ 1999), J. Å. Jönsson (Sweden, 2000-2001); Titular Members: V. A. Davankov (Russia, 1996-2001), J. A. Garcia Dominguez (Spain, 2000-2001), J. Å. Jönsson (Sweden, 1996-1999); Associate Members: D. Berek (Slovakia, 1996-2001), J. A. Garcia Dominguez (Spain, 1998-1999), J. H. Hinshaw (USA, 1996-1999), K. Jinno (Japan, 1995-1999), J. Å. Jönsson (Sweden, 1995), M.-L. Riekkola (Finland, 1996-2001), P. A. Siskos (Greece, $1995-$ 2001): National Representatives: P.S. Anand (India, 1996-1999), J. A. García Domínguez (Spain, 1995-1998), P. Jandera (Czech Republic, 1996-1999), J. Namiesnik (Poland, 1996-2001), A. L. Pires Valente (Brazil, 19951999), D. Pyo (Korea, 1997-2001). 


\begin{abstract}
This paper presents terms and definitions for capillary electromigration techniques for separation and quantitative chemical analysis. Names and descriptions for such techniques (e.g. capillary electrophoresis and capillary electrochromatography) as well as terms for the phenomenon of electroosmotic flow are included.
\end{abstract}

\title{
Introduction
}

Capillary electromigration techniques become increasingly popular and important in chemical analysis, especially in the bioanalytical field. Some of the related terminology can be found in an IUPAC paper on the terminology of electrophoresis in clinical chemistry [1], but these are in many cases not applicable to capillary techniques. The current paper discusses and defines the relevant terms needed in current practice, including names of the various techniques using electromigration principles.

The separations in capillary electromigration techniques are achieved in narrow capillaries by employing a high electric field strength. These techniques include capillary electrophoretic techniques and electrically driven capillary chromatographic techniques, based on different separation principles. However, in some cases, these principles overlap. Capillary electrophoretic techniques have proved to be highly effective for the separation of small organic and inorganic ions, pharmaceuticals, proteins and peptides, DNA and RNA, cells, particles, polymers, explosives, dyes, etc. [2-5]

The presence of electroosmotic flow may contribute to separation even though it is not always needed (e.g. in isoelectric focusing), or may even be completely undesired. The electroosmotic flow originates from the electric double layer on the inner surface of the capillary. For example, in the case of fused silica capillaries, there are three different layers on the surface of the capillary: When the $\mathrm{pH}$ is above 2, the capillary wall becomes negatively charged, as the silanol groups are deprotonated. Overlying this negatively charged surface is a positively charged layer, consisting of an immobile layer, also called a Stern layer or Helmholtz plane, and, finally, a diffuse layer of cations. When an electric field is applied along the capillary, the diffuse layer with a positive net charge moves towards the cathode dragging the solvent within the capillary. This generates a flat flow profile, and as a consequence electroosmotic flow allows high separation efficiencies compared to pressure-driven flow.

Extremely high resolution, short analysis times, the need for a small sample amount, and the low cost of the analysis are advantages obtained by these techniques. In addition, automated commercial CE instruments allow repeatable and reliable operation in routine analyses.

Separation capillaries with internal diameters $20-100 \mu \mathrm{m}$ and lengths of $20-100 \mathrm{~cm}$ are typically used. In the separation of small molecules, uncoated fused silica capillaries are most commonly employed. To prevent surface adsorption of the analytes or matrix components, and to eliminate or control the electroosmotic flow, surface coating or chemical modification of the silanol groups may be necessary. Neutral and charged coatings have been successfully used in a variety of applications. 
Sample injection into the capillary can be performed hydrodynamically by creating a pressure difference between the buffer and the sample solution reservoirs, while dipping the appropriate end of the separation capillary into a sample solution. Using electrokinetic injection, a small injection voltage in applied for a time while the appropriate end of the separation capillary is inserted in the sample reservoir, and the other end in a buffer reservoir.

Ideally, the zone dispersion effects during the separation are due to longitudinal diffusion only. However, temperature gradients inside the capillary may cause additional band broadening effects. The sample introduction technique, the type of sample matrix and adsorption phenomena on the capillary walls may become additional sources of zone dispersion. Temperature control of the separation capillary, the choice of an appropriate buffer medium, a surface treatment technique, the sample type and the way the sample is introduced into the capillary may all be crucial to the success of a particular separation.

Additives such as organic solvents, cyclodextrins, or polymers can be used to control migration and peak shapes of solutes. Cyclodextrins can also be employed as a part of wall coating, rigid gels, and micellar dispersed phases. Capillaries filled with crosslinked gels can be used for the separation of large biomolecules. Incorporation of different chiral selector molecules into such a gel system has been successful in the chiral separations of small molecules. Solutions of entangled linear polymers have partly replaced gels in the separation of biomolecules

In capillary electrochromatography [6,7], columns contain particulate packings used in liquid chromatography or monolithic materials. Coated open tubular columns, similar to gas chromatographic capillary columns can also be employed. Microchannels on a chip-like structure are likely to be exploited more frequently in capillary electromigration techniques after further development of nanotechnology. 


\section{Terms related to capillary electromigration techniques}

Migration time $\left(t_{\mathrm{m}}\right)$

The time required for the analyte to move through the capillary over the distance $(d)$ from the sample introduction point to the detection point.

\section{Electroosmotic flow}

The movement of a liquid in a capillary, generated by the electrical double layer at the capillary inner wall under an electric field.

\section{Electroosmotic hold-up time $\left(t_{\mathrm{eo}}\right)$}

The time required for a liquid in a capillary to move due to electroosmotic flow through the capillary over a distance $(d)$ from the sample introduction point to the detection point.

Note: This time is usually measured as the migration time of a neutral compound (electroosmotic flow marker)

Electroosmotic flow velocity $\left(v_{\mathrm{eo}}\right)$

The velocity of a liquid in a capillary due to electroosmotic flow. It is calculated as $v_{\mathrm{eo}}=d / t_{\mathrm{eo}}$.

Electric field strength (in capillary electromigration techniques) (E)

The applied potential at the sample introduction end of the capillary minus that at the detection end, divided by the total length of the capillary $L_{\text {tot }}$.

Note: $L_{\text {tot }}$ is typically larger than the distance $d$.

Electroosmotic mobility $\left(\mu_{\mathrm{eo}}\right)$

The electroosmotic flow velocity at unit electric field strength $\mathrm{E}$ :

$$
\mu_{\mathrm{eo}}=\frac{\nu_{\mathrm{eo}}}{E}
$$

\section{Electrokinetic potential (or zeta potential) ( $\zeta$ )}

The electric potential difference between the fixed charges on the charged capillary wall and the diffuse charges of the mobile ions. It is related by the Smoluchowski equation [8] to the electroosmotic mobility:

$$
\mu_{\mathrm{eo}}=\frac{\varepsilon \zeta}{4 \pi \eta}
$$

Here, $\varepsilon$ and $\eta$ are the dielectric constant and the absolute viscosity of the electrolyte solution, respectively.

Electrophoretic velocity $\left(v_{\mathrm{ep}}\right)$

The velocity of a charged analyte under the influence of an electric field relative to the background electrolyte. 
Electrophoretic mobility $\left(\mu_{\mathrm{ep}}\right)$

The electrophoretic velocity of an analyte at unit field strength $E$

$$
\mu_{\mathrm{ep}}=\frac{v_{\mathrm{ep}}}{E}
$$

The electrophoretic mobility of an analyte is given by:

$$
\mu_{\mathrm{ep}}=\frac{z F}{f}
$$

In this equation, $z$ is the charge number of the analyte, $F$ is Faraday's constant and the friction coefficient $f$ is described by the Stokes equation

$$
f=6 \pi \eta r
$$

where $r$ is Stoke's radius of the analyte.

Note: also the symbol $\mu$ is used for the friction coefficient [1]. This can cause confusion and is therefore discouraged.

Total velocity of the analyte ( $\left.v_{\text {tot }}\right)$

The sum of the electrophoretic velocity $\left(v_{\mathrm{ep}}\right)$ and the electroosmotic flow velocity $\left(v_{\mathrm{eo}}\right)$, obviously with due regard to signs. $v_{\text {tot }}$ can be experimentally measured as $d / t_{\mathrm{m}}$.

$$
v_{\text {tot }}=v_{\mathrm{ep}}+v_{\mathrm{eo}}
$$

\section{CAPILLARY ELECTROMIGRATION TECHNIQUES}

\section{Capillary Electrophoresis (CE)}

A separation technique carried out in capillaries based solely on the differences in the electrophoretic mobilities of charged species either in aqueous or non-aqueous background electrolyte solutions. These can also contain additives (such as cyclodextrins or polymers) which can interact with the analytes and alter their electrophoretic mobility.

Note: This technique is also known as Capillary Zone Electrophoresis (CZE), but this term is unnecessary and its use is discouraged. 


\section{Capillary Isoelectric Focusing (CIEF)}

An electrophoretic technique for the separation of amphoteric analytes according to their isoelectric points by the application of an electric field along a $\mathrm{pH}$ gradient formed in a capillary.

\section{Capillary Sieving Electrophoresis (CSE)}

An electrophoretic separation technique, which takes place in a capillary filled with a sieving medium. The separation is based on differences in size and shape of the charged analytes.

\section{Capillary Gel Electrophoresis (CGE)}

A special case of CSE when the capillary is filled with a gel.

\section{Capillary Isotachophoresis (CITP)}

An electrophoretic separation technique in a discontinuous buffer system in which the analytes migrate according to their electrophoretic mobilities, forming a chain of adjacent zones moving with equal velocity between two solutions, leading and terminating electrolytes, bracketing the mobility range of the analytes.

\section{Affinity Capillary Electrophoresis (ACE)}

An electrophoretic separation technique involving biospecific interactions between the analyte and a substance present in the electrolyte solution.

\section{Electrokinetic Capillary Chromatography (ECC)}

A separation technique based on a combination of electrophoresis and interactions of the analytes with additives (e.g. surfactants), which form a secondary phase moving at a different velocity. In order to achieve separation either the analytes or the secondary phase should be charged.

Note: This is not totally compatible with the definition of chromatography.

\section{Micellar Electrokinetic Capillary Chromatography (MECC)}

A special widely used case of ECC in which the secondary phase is a micellar dispersed phasecapillary.

Note: The same technique is sometimes called Micellar Electrokinetic

Chromatography (MEKC)

Migration time of the micelles, $\left(t_{\mathrm{mc}}\right)$

Experimentally determined as the migration time of an analyte that is completely retained in the micellar phase. 
Partition ratio in $M E C C, k_{\text {mecc }}$

Defined as:

$$
k_{\mathrm{mecc}}=\frac{n_{\mathrm{mc}}}{n_{\mathrm{aq}}}
$$

where $n_{\mathrm{mc}}$ and $n_{\mathrm{aq}}$ are the amounts of the analyte in the micellar and aqueous phases, respectively.

Note: In the case of an electrically neutral analyte, $k_{\text {mecc }}$ can be calculated directly from the migration times:

$$
k_{\mathrm{mecc}}=\frac{\left(t_{\mathrm{m}}-t_{\mathrm{eo}}\right)}{t_{\mathrm{eo}}\left(1-t_{\mathrm{m}} / t_{\mathrm{mc}}\right)}
$$

Note: $k_{\text {mecc }}$ should not be confused with the retention factor $k$ in chromatography, as those are different quantities.

\section{Microemulsion electrokinetic capillary chromatography (MEECC)}

A special technique of ECC where a microemulsion is employed as the dispersed phase.

Note: The equations 7-8 are valid also in MEECC, with the symbol $k_{\text {mecc }}$ being replaced with $k_{\text {meecc. }}$.

\section{Capillary Electrochromatography (CEC)}

CEC is a separation technique in which the mobile phase movement through a capillary, filled, packed or coated with a stationary phase, is achieved by electroosmotic flow (which may be assisted by pressure). The retention is due to a combination of electrophoretic migration and chromatographic retention. 


\section{Symbols}

$k_{\text {mecc }}$ Partition ratio in micellar electrokinetic capillary chromatography

$k_{\text {meecc }}$ Partition ratio in microemulsion electrokinetic capillary chromatography)

$v_{\mathrm{eo}} \quad$ Electroosmotic flow velocity

$v_{\mathrm{ep}} \quad$ Electrophoretic velocity

$v_{\text {tot }} \quad$ Total velocity of the analyte

$t_{\text {eo }} \quad$ Electroosmotic hold-up time

$t_{\mathrm{m}} \quad$ Migration time of the analyte

$t_{\mathrm{mc}} \quad$ Migration time of the micelles

$E \quad$ Electric field strength

$\zeta \quad$ Zeta potential

$\varepsilon \quad$ Dielectric constant

$\eta \quad$ Absolute viscosity

$\mu_{\mathrm{eo}} \quad$ Electroosmotic mobility

$\mu_{\mathrm{ep}} \quad$ Electrophoretic mobility

$z \quad$ Ion charge number

F Faraday's constant

$f \quad$ Friction coefficient

$r \quad$ Stoke's radius

\section{References}

1. G. Férard, Quantities and Units for Electrophoresis in the Clinical Laboratory. Pure Appl. Chem. 66, 891-896 (1994).

2. P. Camillari, Capillary Electrophoresis ( $2^{\text {nd }}$ ed), CRC Press, Boca Raton, FL, 1998

3. D. E. Baker, Capillary Electrophoresis, John Wiley and Sons, New York, 1995

4. M.G. Khaledi, High-Performance Capillary Electrophoresis, John Wiley and Sons, New York, 1998

5. W. Kok, Capillary Electrophoresis: Instrumentation and operation, Chromatographia Supplement Vol 51, 2000

6. Z. Deyl, F. Svec, Capillary Electrochromatography, Elsevier Sci., Amsterdam, 2001

7. K. Bartle, P. Myers, Capillary Electrochromatography, Royal Society of Chemistry, London, 2001

8. M. Smoluchowski, Handbuch der Elektrizität und des Magnetismus, Vol.2, Leipzig, Germany, 1914, p. 366 